\title{
Deep Inelastic Scattering with the SPS Muon Beam
}

\author{
Gerhard K. Mallot ${ }^{1}$ and Rüdiger Voss ${ }^{2}$ \\ CERN, CH-1211 Geneva 23, Switzerland \\ 11gerhard.mallot@cern.ch \\ ${ }^{2}$ ruediger.voss@cern.ch
}

\begin{abstract}
We review results from deep inelastic muon scattering experiments at the SPS which started in 1978, and are still actively pursued today. Key results include the precision measurement of scaling violations and of the strong coupling constant, spin-dependent structure functions, and studies of the internal spin structure of protons and neutrons. These experiments have revealed a wealth of details about the internal structure of nucleons in terms of quarks and gluons.
\end{abstract}

\section{Introduction}

At the 14th International Conference in High Energy Physics in Vienna in 1968, SLAC reported for the first time the "scaling" behaviour of the electron-nucleon cross-section in the deep inelastic continuum, and W. K. H. Panofsky remarked that "... theoretical speculations are focused on the possibility that these data might give evidence on the behaviour of point-like, charged structures within the nucleon." 1 Soon after, it was realised that the parton structure of the nucleon discovered by the first electron-nucleon scattering experiments in the deep inelastic regime indeed confirmed the quark model of Gell-Mann ${ }^{2}$ and Zweig. ${ }^{3}$

The early SLAC results on the quark-parton structure of the nucleon had a profound impact on the first-generation experimental programme of the CERN Super Proton Synchrotron (SPS), and most notably on the muon and neutrino scattering experiments. Several groups realised the potential of this new machine to extend the landmark SLAC experiments much 'deeper' into the inelastic regime by building a high-intensity, high-energy muon beam. This was the beginning of one of the most prolific fixed-target physics programmes of CERN that started in 1978, soon after the commissioning of the SPS, and is still vigorously pursued today.

This brief review focuses on two central components of this programme which, from a present-day perspective, have had the most lasting impact: (a) the precision measurement of scaling violations for tests of perturbative QCD and measurements of the strong coupling constant, and (b) the measurement of spin-dependent structure functions, the discovery of the 'spin crisis', and comprehensive studies of the spin structure of the nucleon. However, it must not be overlooked that the CERN muon programme has, over the years, produced a wealth of other, sometimes 
unexpected results. Examples are the discovery of nuclear effects in deep inelastic scattering, ${ }^{4}$ the first observation of weak-electromagnetic interference effects in muon scattering, or measurements of charm production.

\section{Beam and Detectors}

The SPS muon beam M2 ${ }^{5}$ was first commissioned in 1978 and is still in operation today, with only minor modifications. It is likely to be the best and most versatile high-energy muon beam ever designed, combining a wide range of momenta up to $300 \mathrm{GeV}$ with high intensities and minimal halo background. The beam has a natural longitudinal polarisation that can be tuned by varying the momentum ratio of decay muons to parent pions, and can reach values up to $\approx 80 \%$. A high beam polarisation is an essential prerequisite for the measurement of spin-dependent structure functions.

\subsection{Early detectors}

Two large detectors were built for the first generation of experiments, the NA2 experiment of the European Muon Collaboration (EMC) ${ }^{6-8}$ and the NA4 experiment of the Bologna-CERN-Dubna-Munich-Saclay (BCDMS) Collaboration. ${ }^{9,} 10$ The two collaborations choose radically different, complementary experimental approaches. The EMC detector was a conventional open-geometry spectrometer built around a large air-gap dipole magnet, instrumented with proportional and drift chambers for particle tracking. The main advantages of this design were an excellent momentum resolution, a large kinematic range, and the ability to partly resolve the hadronic final state of the deep inelastic interaction. A disadvantage was the maximum target length allowed by the spectrometer layout, of order $1 \mathrm{~m}$, which limits the statistical accuracy of many measurements.

In contrast, the BCDMS spectrometer was specifically designed for the inclusive measurement of high-momentum final state muons. It was based on a large, modular toroidal iron magnet of $50 \mathrm{~m}$ length instrumented with multiwire proportional chambers. In the centre, the toroid contained a modular target of almost the same length that could be filled with liquid hydrogen or deuterium, or replaced by solid target material. Principal advantages of this design were the enormous luminosity and the excellent muon identification through immediate absorption of the hadronic shower, which could not be resolved by the detector. Another obvious drawback was the comparatively poor momentum resolution due to multiple scattering in the iron magnet, limited to $\Delta p / p \approx 10 \%$ over most of the momentum range.

The EMC and BCDMS experiments took data from 1978 until 1985, both with liquid hydrogen, liquid deuterium, and solid nuclear targets. In addition, EMC made first measurements with a polarised solid ammonia target. Whereas the BCDMS spectrometer was subsequently dismantled, the more versatile EMC spectrometer 
underwent several upgrades, in particular for the later NMC (NA37, 1986-1989) and SMC (NA47, 1992-1996) experiments. The NMC Collaboration (where the N stands for "New") refined and improved the EMC measurements of unpolarised structure functions, with a strong focus on the study of nuclear effects with a variety of heavy targets. ${ }^{11}$ The SMC experiment (where the S stands for "Spin", obviously) was devoted exclusively to polarised muon-nucleon scattering with solid butanol, deuterated butanol, and ammonia targets.

\subsection{The COMPASS detector}

The most comprehensive rebuilt of the EMC/NMC/SMC spectrometer was undertaken by the COMPASS (NA58) Collaboration, which today continues the successful tradition of muon scattering at CERN, and still uses some of the original EMC equipment. The COMPASS experiment ${ }^{12}$ started taking data in 2002.

Contrary to the one-stage $\mathrm{EMC}^{6}$ and SMC spectrometers, the COMPASS detector (Fig. 1) is a two-stage magnetic spectrometer with the SM1 and SM2 dipoles. This results in a very large acceptance which is important for semi-inclusive deep inelastic scattering (SIDIS) experiments. Other essential additions and improvements concern the particle identification detectors, the large-acceptance, superconducting target magnet, and last but not least the high rate and data acquisition capabilities, which went up from the order of $100 \mathrm{~Hz}$ to $25 \mathrm{kHz}$.

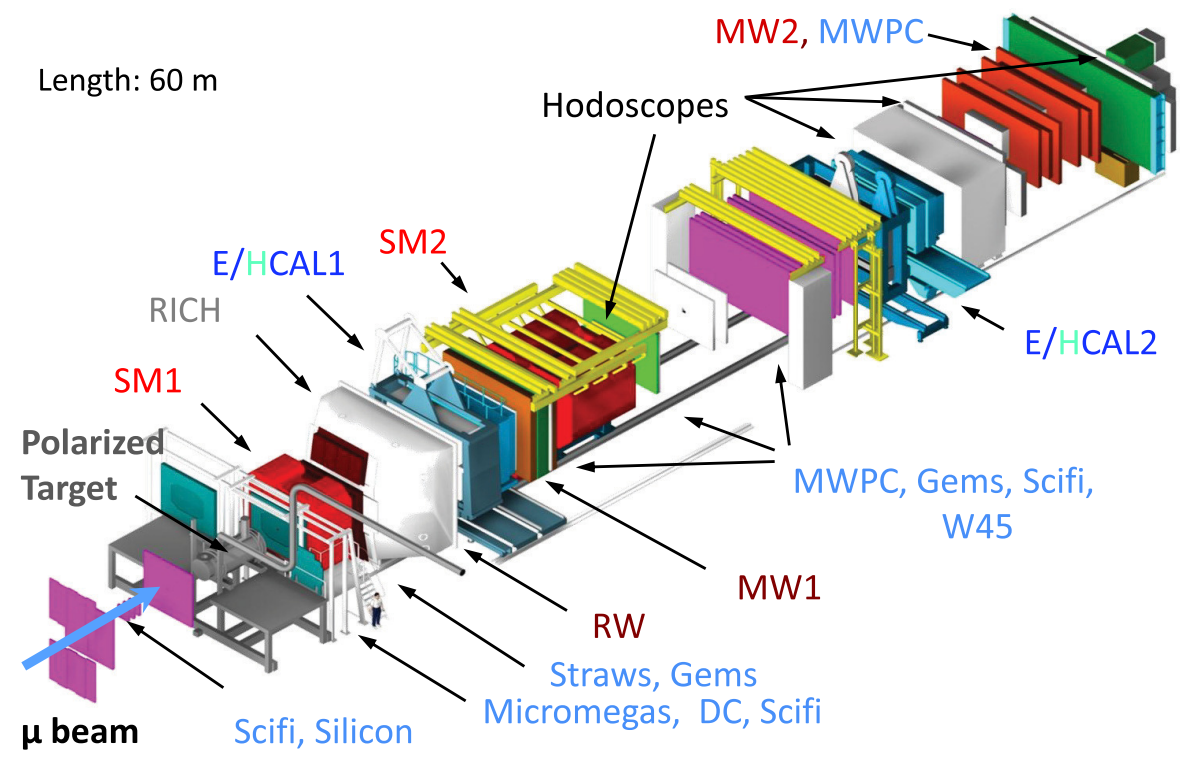

Fig. 1. Artist's view of the COMPASS spectrometer. For a description see the text. 
The COMPASS spectrometer is installed in the M2 muon beam line delivering muons of $160-200 \mathrm{GeV}$ with a polarisation of about $80 \%$. The usable beam intensity is typically $2 \times 10^{7} / \mathrm{s}$ during a $9.6 \mathrm{~s}$ long spill. The repetition rate varies and is typically about $1 / 40 \mathrm{~s}$. The momentum of each beam muon is measured in the beam momentum station.

Charged particles are tracked in the beam region by scintillating fibre stations (SciFi) and silicon detectors. In the region close to the beam, micromega and gas-electron-multiplier (GEM) gaseous detectors with high rate capabilities are deployed. The backbone of tracking in the intermediate region is formed by multiwire proportional chambers (MWPCs). Finally, the large area tracking is covered by drift chambers (DC, W45) and drift tubes (Straws, RW, MW).

The velocity of charged particles is measured in a ring-imaging Cherenkov detector $(\mathrm{RICH})$, which can separate pions and kaons from $9 \mathrm{GeV}$ up to $50 \mathrm{GeV}$. The photon detector comprises multianode-photomultiplier tubes and in the periphery MWPCs with photosensitive CsI cathodes. The energy of charged particles is measured in sampling hadron calorimeters (HCAL), while neutral particles, in particular high-energy photons, are detected in electromagnetic calorimeters (ECAL).

Event recording is triggered by the scattered muon, which is "identified" by its ability to traverse thick hadron absorbers located just upstream of the Muon Wall detectors (MW), and detected by various systems of scintillator hodoscopes. The same spectrometer is also used for an experimental programme on hadron spectroscopy using pion, kaon and proton beams. ${ }^{13}$

\subsection{The COMPASS polarised target}

The heart of the experiment is the superconducting polarised target system. It comprises a $2.5 \mathrm{~T}$ solenoid and a $0.6 \mathrm{~T}$ dipole magnet, a ${ }^{3} \mathrm{He} /{ }^{4} \mathrm{He}$ dilution refrigerator originating from $\mathrm{SMC}$, a $70 \mathrm{GHz}$ microwave system for the dynamic nuclear polarisation (DNP), and an NMR system to measure the target polarisation. The target material is cooled down to about $60 \mathrm{mK}$ in frozen spin mode. Irradiated ammonia $\left(\mathrm{NH}_{3}\right)$ and lithium-6 deuteride $\left({ }^{6} \mathrm{LiD}\right)$ were selected as proton and deuteron targets, respectively. Typical polarisations achieved are $85 \%$ for protons and $50 \%$ for deuterons. The target volume has an overall length of $1.3 \mathrm{~m}$ and comprises two or three cells with opposite polarisations. The target spins are rotated typically once per day by rotation of the magnetic field vector. The rotation can be stopped in transverse position for measurements with transverse target polarisation. For such measurements the polarisation is inverted typically once per week by DNP.

\section{Unpolarised Nucleon Structure Functions}

Deep inelastic lepton-nucleon scattering is loosely defined as scattering at energy transfers much larger than the parton binding energy in the nucleon, such that the interaction occurs at the parton level and thus probes the internal quark-parton 


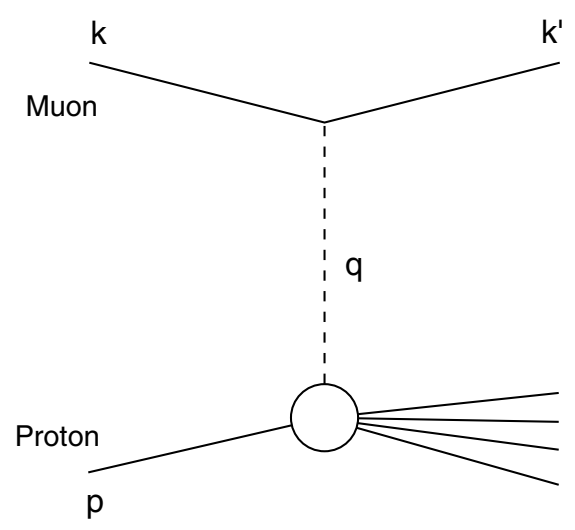

Fig. 2. Deep inelastic muon-nucleon scattering in lowest order.

structure of the target nucleon. Charged lepton scattering can be mediated through neutral-current $\gamma$ or $Z$ exchange, or through charged current $W^{ \pm}$exchange. At typical SPS fixed-target energies of a few hundred $\mathrm{GeV}$, it is dominated by single photon exchange (Fig. 2). Consequently, muon scattering at CERN has mostly focused on this channel. The excellent luminosity of the BCDMS spectrometer has allowed for measurements of $\gamma-Z$ interference ${ }^{14,15}$ which however have been completely superseded by data from HERA, and are not reviewed here.

\subsection{Cross-section and structure functions}

For inclusive scattering where the scattering amplitudes are summed over all possible hadronic final states, the unpolarised cross-section can be written as a function of two independent kinematic variables. One usually chooses two of the following Lorentz invariant variables,

- the squared four-momentum transfer

$$
Q^{2}=-q^{2}=-\left(k-k^{\prime}\right)^{2}=4 E E^{\prime} \sin ^{2} \theta ;
$$

- the energy transfer to the hadronic system

$$
\nu=\frac{p \cdot q}{M}=E^{\prime}-E
$$

- the Bjorken scaling variable

$$
x=\frac{Q^{2}}{2 p \cdot q}=\frac{Q^{2}}{2 M \nu}
$$

- and the scaling variable

$$
y=\frac{p \cdot q}{p \cdot k}=\frac{\nu}{E}
$$


In these equations, $k, k^{\prime}, p$ and $q$ are the four-vectors of the initial and final state lepton, the target nucleon, and the exchanged boson. $M$ is the mass of the target nucleon and the lepton mass has been neglected. $E, E^{\prime}$, and $\theta$ are the energies of the incident and scattered lepton, and the lepton scattering angle, in the laboratory frame.

The differential cross-section for unpolarised deep inelastic charged lepton scattering can be written, in the Born approximation, as ${ }^{16,17}$

$$
\frac{d^{2} \sigma}{d Q^{2} d x}=\frac{4 \pi \alpha^{2}}{Q^{4}} \frac{1}{x}\left[x y^{2} F_{1}\left(x, Q^{2}\right)+\left(1-y-\frac{M x y}{2 E}\right) F_{2}\left(x, Q^{2}\right)\right],
$$

where $\alpha$ is the electromagnetic coupling constant and $F_{1}\left(x, Q^{2}\right)$ and $F_{2}\left(x, Q^{2}\right)$ are the unpolarised structure functions of the nucleon,

$$
\begin{aligned}
& F_{1}\left(x, Q^{2}\right)=\frac{1}{2 x} \sum_{i} e_{i}^{2} x q_{i}\left(x, Q^{2}\right), \\
& F_{2}\left(x, Q^{2}\right)=2 x F_{1}\left(x, Q^{2}\right)=\sum_{i} e_{i}^{2} x q_{i}\left(x, Q^{2}\right) .
\end{aligned}
$$

In these expressions, $q_{i}\left(x, Q^{2}\right)$ is the probability distribution of partons of flavour $i$ in the kinematic variables $x$ and $Q^{2}$ and the index $i$ runs over the active parton flavours in the nucleon. The SLAC discovery that the structure functions depend, at least approximately, on the dimensionless scaling variable $x$ only, ${ }^{18,} 19$

$$
q_{i}\left(x, Q^{2}\right) \approx q_{i}(x)
$$

— the effect commonly referred to as "scaling" — is interpreted in the Quark-Parton Model (QPM) as elastic scattering on dimensionless, i.e. pointlike scattering centres inside the nucleon. Scaling becomes exact in the Bjorken limit where $Q^{2}, \nu \rightarrow \infty$ at constant $x,{ }^{20}$ such that the transverse momentum of partons in the infinite momentum frame of the proton becomes negligible.

\section{2. $\quad$ Scaling violations}

When the muon experiments at the SPS started taking data in 1978, scaling and the QPM were well established experimentally and phenomenologically. The key interest of the experiments shifted soon to the measurement of small deviations from exact scaling behaviour, or scaling violations. As an example, the most representative fixed-target measurements of the proton structure function $F_{2}^{p}\left(x, Q^{2}\right)$ are shown in Fig. 3. ${ }^{\mathrm{a}}$ They exhibit a characteristic rise of the structure function with $Q^{2}$ at small $x$, a decrease at large $x$, and "apparent scaling" at $x \approx 0.15$.

\footnotetext{
${ }^{a}$ The first-generation data from muon scattering at the SPS were plagued by significant disagreements between the EMC and BCDMS results on $F_{2}$. The NMC Collaboration later remeasured this structure function with the upgraded EMC spectrometer, and eventually confirmed the BCDMS results.
} 

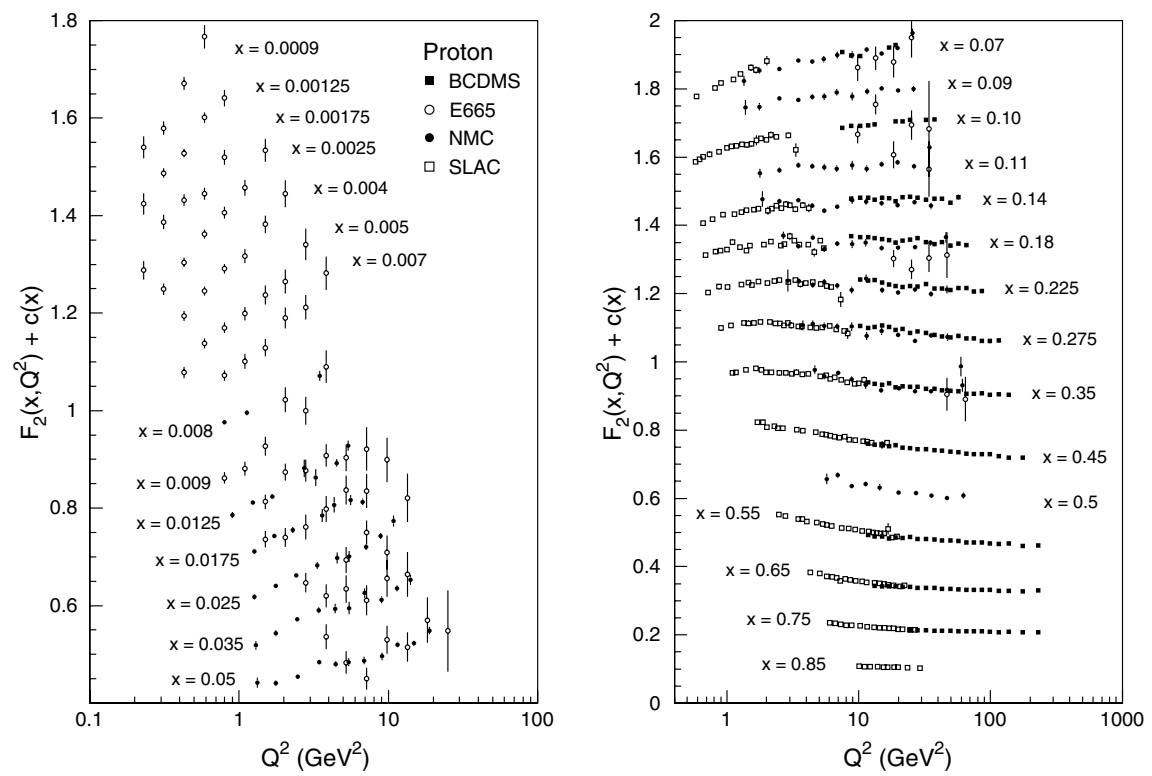

Fig. 3. The proton structure function $F_{2}^{p}$ measured in deep inelastic muon scattering by the BCDMS $^{21}$ and $\mathrm{NMC}^{22}$ experiments, shown as a function of $Q^{2}$ for bins of fixed $x$. The CERN data are complemented at small $Q^{2}$ by the SLAC electron scattering data, ${ }^{23}$ and at small $x$ by muon scattering data from the Fermilab E665 experiment. ${ }^{24}$ Only statistical errors are shown. For the purpose of plotting, a constant $c(x)=0.1 i_{x}$ is added to $F_{2}^{p}$ where $i_{x}$ is the number of the $x$ bin, ranging from $1(x=0.05)$ to $14(x=0.0009)$ on the left-hand figure, and from $1(x=0.85)$ to $15(x=0.007)$ on the right-hand figure.

\subsection{Tests of perturbative $Q C D$}

Scaling violations occur naturally in Quantum Chromodynamics since, at large parton momenta $x$ and increasing $Q^{2}$, the structure functions are increasingly depleted by hard gluon radiation from quarks; at small $x$, they are enriched by gluon conversion into low-momentum quark-antiquark pairs. The initial years of experimentation with the SPS muon beam coincided with the emergence of QCD as the universally accepted theory of the strong interaction, and were an active and exciting period of cross-fertilisation of phenomenology and experiments. Precise data on scaling violations turned out to be one of the most powerful tools to test the perturbative branch of the new theory, and allowed for one of the best early measurements of the strong coupling constant.

The $Q^{2}$ evolution of the strong coupling constant $\alpha_{s}$ is controlled by the renormalisation group equation of QCD. The "canonical" - but by no means unique - solution usually adopted for the analysis of deep inelastic data is, in next-to-leading order (NLO),

$$
\alpha_{s}\left(Q^{2}\right)=\frac{4 \pi}{\beta_{0} \ln \left(Q^{2} / \Lambda^{2}\right)}\left[1-\frac{\beta_{1}}{\beta_{0}^{2}} \frac{\ln \ln \left(Q^{2} / \Lambda^{2}\right)}{\ln \left(Q^{2} / \Lambda^{2}\right)}\right]
$$


where the so-called beta functions are given by

$$
\beta_{0}=11-\frac{2}{3} N_{f}, \quad \beta_{1}=102-\frac{38}{3} N_{f}
$$

and $N_{f}$ is the effective number of active quark flavours in the scattering process. The parameter $\Lambda$ is the so-called "mass scale" of QCD and has the physical meaning of a typical energy at which the running coupling constant (9) becomes large and the perturbative expansion breaks down. Its value is not predicted by QCD and can only be determined by experiment. Since $\alpha_{s}$ is the physical observable, the numerical value of $\Lambda$ depends on $N_{f}$ and, beyond leading order, on the renormalisation scheme assumed to compute the perturbative QCD expansions.

The $Q^{2}$ evolution of the effective quark and gluon distribution is predicted by the Altarelli-Parisi equations, ${ }^{25}$

$$
\begin{aligned}
\frac{d q^{N S}\left(x, Q^{2}\right)}{d \ln Q^{2}} & =\frac{\alpha_{s}\left(Q^{2}\right)}{2 \pi} \int_{x}^{1} q^{N S}\left(t, Q^{2}\right) P^{N S}\left(\frac{x}{t}\right) \frac{d t}{t}, \\
\frac{d q^{S I}\left(x, Q^{2}\right)}{d \ln Q^{2}} & =\frac{\alpha_{s}\left(Q^{2}\right)}{2 \pi} \int_{x}^{1}\left[q^{S I}\left(t, Q^{2}\right) P_{q q}\left(\frac{x}{t}\right)+C_{q} g\left(t, Q^{2}\right) P_{q g}\left(\frac{x}{t}\right)\right] \frac{d t}{t}, \\
\frac{d g\left(x, Q^{2}\right)}{d \ln Q^{2}} & =\frac{\alpha_{s}\left(Q^{2}\right)}{2 \pi} \int_{x}^{1}\left[g\left(t, Q^{2}\right) P_{g g}\left(\frac{x}{t}\right)+C_{g} q^{S I}\left(t, Q^{2}\right) P_{g q}\left(\frac{x}{t}\right)\right] \frac{d t}{t},
\end{aligned}
$$

where $S I$ and $N S$ denote flavour singlet and non-singlet combinations of quark distributions, respectively, $g$ is the gluon distribution, and the $C_{i}$ are a set of coefficients. $P^{N S}, P_{q q}$, etc. are so-called splitting functions describing the QCD diagrams which can be calculated in perturbative QCD as power series in $\alpha_{s}$.

\subsection{Measurement of the strong coupling constant}

The $F_{2}$ measurements of BCDMS at large $x$ and $Q^{2}$ with carbon, hydrogen, and deuterium targets were the first high statistics data that yielded a conclusive determination of $\Lambda_{\mathrm{QCD}} \cdot{ }^{26-28}$ The original BCDMS fits to the hydrogen and deuterium data were later superseded by a careful analysis by Virchaux and Milsztajn of the combined SLAC and BCDMS hydrogen and deuterium data. ${ }^{29}$ Since the SLAC data extend down to four-momentum transfers as low as $Q^{2}=1 \mathrm{GeV}^{2}$, these authors make an allowance for non-perturbative "higher twist" contributions to the observed scaling violations at small $Q^{2}$. These higher twist effects are mostly due to longdistance final state interactions which are difficult to calculate in perturbative QCD and there is little theoretical prejudice about their kinematical dependence except that they can be expanded into power series in $1 / Q^{2} .{ }^{30}$ This suggests an ansatz

$$
F_{2}\left(x, Q^{2}\right)=F_{2}^{L T}\left(x, Q^{2}\right)\left[1+\frac{C_{H T}(x)}{Q^{2}}\right]
$$


where the leading twist structure function $F_{2}^{L T}$ follows the Altarelli-Parisi equations, and which gives indeed a very satisfactory fit to the data (Fig. 4). The quality
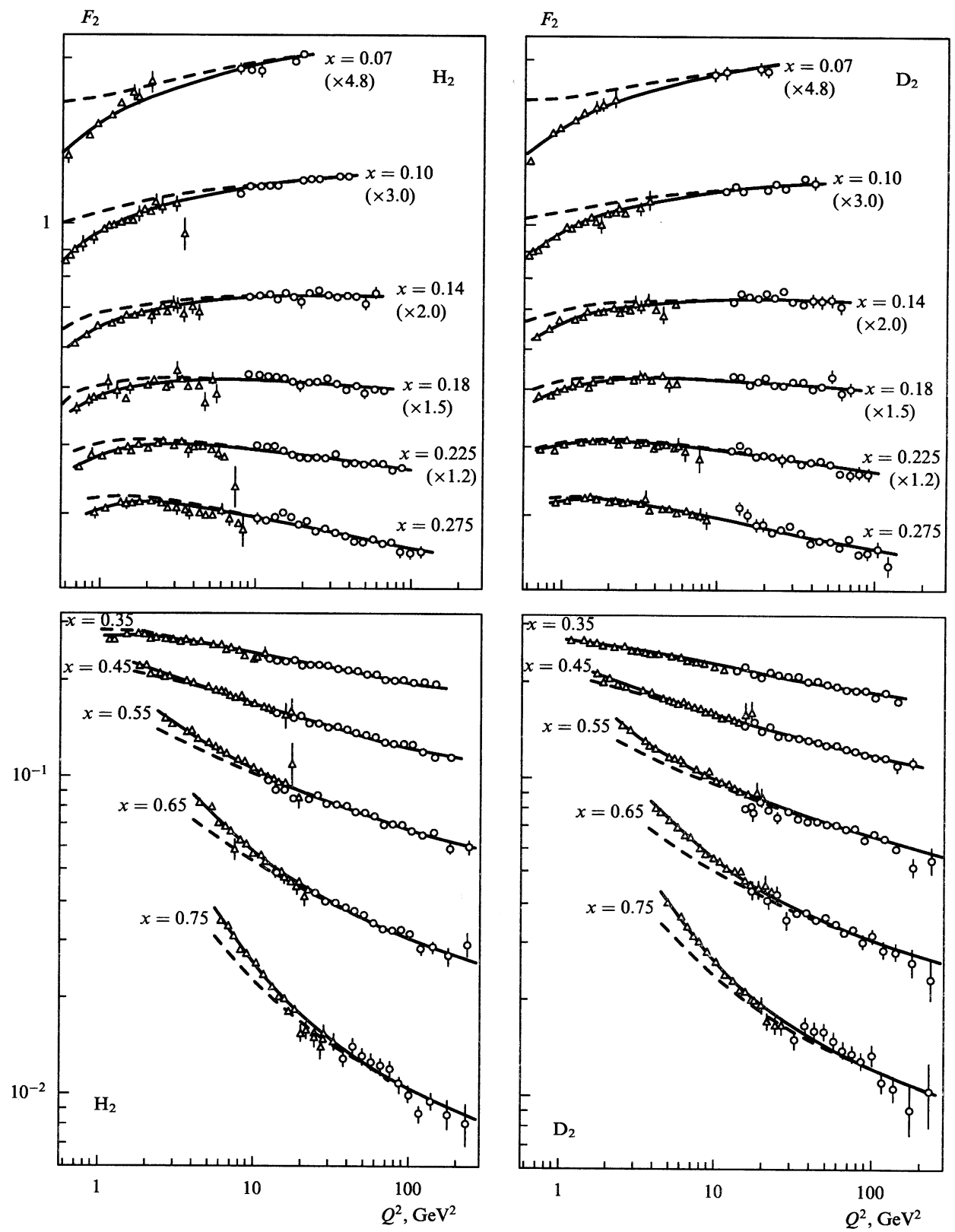

Fig. 4. QCD fit to the SLAC (triangles) and BCDMS (circles) data on $F_{2}\left(x, Q^{2}\right)$. The dashed line is the purely perturbative fit with the leading twist structure function $F_{2}^{L T}\left(x, Q^{2}\right)$. The solid line includes the higher twist contribution discussed in the text. 


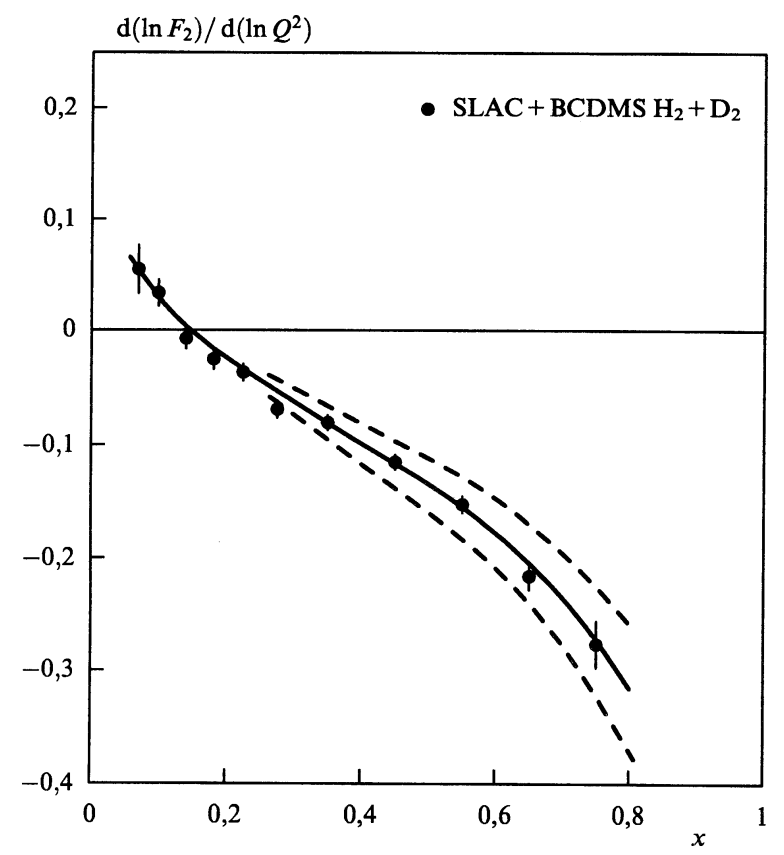

Fig. 5. Scaling violations $d \ln F_{2} / d \ln Q^{2}$ observed in the combined SLAC/BCDMS hydrogen and deuterium data. Errors are statistical only. The solid line is a QCD fit corresponding to $\alpha_{s}\left(M_{Z}^{2}\right)=$ $0.113 ;$ the dashed lines correspond to $\Delta \alpha_{s}\left(M_{Z}^{2}\right)=0.010$.

of the fit is best illustrated in the representation of the "logarithmic slopes" which shows the derivative of the structure function with respect to $\ln Q^{2}$ as predicted by the Altarelli-Parisi equations, averaged over the $Q^{2}$ range of each bin in $x$ (Fig. 5). In this analysis, the higher twist term $C_{H T}(x)$ of Eq. (13) is fitted by a set of constants in each bin of $x$. These coefficients are compatible with zero for $x<0.4$, i.e. perturbative QCD can describe scaling violations in this region down to $Q^{2}$ as small as $1 \mathrm{GeV}^{2}$.

In the same analysis, Virchaux and Milsztajn have also estimated the "theoretical" uncertainty due to the neglect of higher order terms in the perturbative QCD expansions. Their final result for $\alpha_{s}$ at $Q^{2}=M_{Z}^{2}$ is

$$
\alpha_{s}\left(M_{Z}^{2}\right)=0.113 \pm 0.003 \text { (exp.) } \pm 0.004 \text { (theor.). }
$$

A later analysis by Alekhin ${ }^{31}$ based on the combined SLAC, BCDMS, and NMC data yielded $\alpha_{s}\left(M_{Z}^{2}\right)=0.1183 \pm 0.0021$ (exp.) \pm 0.0013 (theor.). These data still provide significant input to the present world average of $\alpha_{s}$ from deep inelastic scattering, and are in good agreement with the final combined result from LEP. ${ }^{32}$

As a byproduct, these QCD fits can also provide an estimate of the gluon distribution in the nucleon (Eq. (11)). Since the gluon distribution is strongly peaked at small $x$, however, this estimate is now superseded by fits to more recent data, in particular data from HERA, with better coverage of this kinematic region. 


\section{Nucleon Spin and Polarised Deep Inelastic Scattering}

One of the last experiments performed by the EMC was in 1985 the measurement of the double-spin cross-section asymmetry for inclusive deep inelastic scattering of longitudinally polarised muons off longitudinally polarised protons. As in the unpolarised case initial measurements had been performed at SLAC at lower energies in a limited kinematic range. While the SLAC measurements were in line with expectations from the QPM, the EMC results showed in the previously unmeasured small- $x$ region $(x<0.1)$ a clear disagreement with potentially dramatic consequences. $^{33,34}$ In the QPM, the nucleon spin is supposed to arise entirely from the quark spins, while in relativistic quark models a contribution of about $60 \%$ is expected. However, the EMC result was compatible with zero. Leader and Anselmino conclude in $1988^{35}$ in an article entitled "A crisis in the parton model: where, oh where is the proton's spin?":

(a) Orbital angular momentum may be important; and this is perfectly consistent with what is known about the intrinsic $k_{T}$ [transverse momentum] of quarks.

(b) The sacrosanct Bjorken sum rule may be broken. A measurement of $g_{1}^{n}$ [of the neutron] is clearly now vital!

(c) The experiment may be wrong. Given its fundamental importance it should be redone, ... obviously, with great emphasis on the small $x$ region.

The unexpected result, dubbed the spin crisis, gave birth to many new experiments, including those of the Spin Muon (SMC) and COMPASS Collaborations at CERN. A very fruitful interplay between theory and experiment enrolled opening up a whole field of research now extending to transverse polarisation, transverse-momentum dependent (TMD) and generalised parton distributions (GPD). A comprehensive recent review is presented in Ref. 36 including non-CERN work by HERMES at DESY, Jefferson Lab and the Relativistic Heavy Ion Collider (RHIC) at the Brookhaven National Laboratory not covered here.

\subsection{Longitudinal spin}

The spin of the nucleon of $1 / 2$ (in units of $\hbar$ ) can be decomposed into contributions from spins ' $\Delta$ ' and orbital angular momenta $L$ from both quarks $q$ and gluons $g$

$$
\frac{1}{2}=\frac{1}{2} \Delta \Sigma+\Delta g+L_{g}+L_{q}
$$

with

$$
\Delta \Sigma=\Delta u+\Delta d+\Delta s+\text { aq. }
$$

where "aq." indicates the corresponding terms for antiquarks. The individual spin contributions from the up, down, and strange quarks to the nucleon spin are given 
by the first moments $\Delta u, \Delta d$, and $\Delta s$ of the corresponding helicity distributions $\Delta q_{i}(x)$

$$
\Delta i=\int_{0}^{1} \Delta q_{i}(x) \mathrm{d} x \quad \text { with } i=u, d, s, \text { and antiquarks }
$$

where

$$
\Delta q_{i}(x)=q_{i}^{+}(x)-q_{i}^{-}(x) .
$$

Here, the superscripts + and - denote the helicity of the quarks; the gluon helicity distribution $\Delta g(x)$ is defined accordingly. While in the unpolarised case the sum $q_{i}(x)=q_{i}^{+}(x)+q_{i}^{-}(x)$ of the number densities of quarks appears, in the polarised case this role is taken by their difference.

The quark helicity distributions $\Delta q_{i}\left(x, Q^{2}\right)$ in the nucleon can be accessed via the spin-dependent structure function $g_{1}\left(x, Q^{2}\right)$, which appears in the DIS crosssection. In the QPM the structure function $g_{1}$ is given by

$$
g_{1}(x)=\frac{1}{2} \sum_{i} e_{i}^{2} \Delta q_{i}(x)
$$

where $e_{i}$ denotes the electric charge of the struck quark (compare Eq. (6)). Like $F_{1}$, also $g_{1}$ depends on Bjorken $x$ and logarithmically on $Q^{2}$.

\subsubsection{Sum rules}

For the proton the first moment $\Gamma_{1}$ of $g_{1}$ can be decomposed into three axial charges: the isovector charge $a_{3}$, the octet charge $a_{8}$ and the flavour-singlet charge $a_{0}$

$$
\Gamma_{1}^{p}\left(Q^{2}\right)=\int_{0}^{1} g_{1}^{p}\left(x, Q^{2}\right) \mathrm{d} x=\frac{1}{12}\left(a_{3}+\frac{1}{3} a_{8}\right)+\frac{1}{9} a_{0} .
$$

They are given in terms of flavour contributions by

$$
a_{3}=\Delta u-\Delta d+\text { aq. }, \quad a_{8}=\Delta u+\Delta d-2 \Delta s+\text { aq. }, \quad a_{0}=\Delta u+\Delta d+\Delta s+\text { aq. }
$$

The isovector and isoscalar charges come with $Q^{2}$-dependent Wilson coefficients, which are calculable in perturbative QCD and are omitted here. For the $Q^{2}$ dependent flavour-singlet axial charge $a_{0}$ usually its normalisation-scheme independent value at $Q^{2} \rightarrow \infty$ is quoted. In the $\overline{\mathrm{MS}}$ renormalisation scheme, $a_{0}$ is identical to $\Delta \Sigma$, the sum of all quark spins (Eq. (15)). However, while $a_{0}$ is an observable, $\Delta \Sigma$ per se is not. The isovector axial charge $a_{3}$ is equal to the weak coupling constant $\left|g_{A} / g_{V}\right|$ measured independently in neutron decay and $a_{8}$ is known from hyperon decays assuming $\mathrm{SU}(3)$ flavour symmetry. Both are $Q^{2}$ independent.

Subtracting from Eq. (19) the corresponding equation for the neutron yields the fundamental Bjorken sum rule 37,38 which for $Q^{2} \rightarrow \infty$ reads

$$
\Gamma_{1}^{p}-\Gamma_{1}^{n}=\frac{1}{6}\left|\frac{g_{A}}{g_{V}}\right|
$$


The $a_{0}$ and $a_{8}$ axial charges cancel in the difference of proton and neutron, i.e. when $\Delta u$ is replaced by $\Delta d$ and vice versa. This famous sum rule links the first moment of the structure function $g_{1}$ (for $Q^{2} \rightarrow \infty$ ) to the neutron decay constant and was derived already in 1966 using current algebra. However, Bjorken first dismissed it as a 'worthless' equation, because performing a measurement with a polarised neutron target seemed impossible at the time. Only three years later he reconsidered this statement "in light of the present experimental and theoretical situation". It took until 1992 that the first neutron (deuteron and helium-3) measurements were performed. Earlier proposals to measure the neutron as part of the E130 experiment at SLAC were finally not carried out. At this time the proton results were in line with expectations and thus a neutron measurement was less pressing.

In 1973, Ellis and Jaffe used Eq. (19) to make a prediction ${ }^{39}$ for $\Gamma_{1}$ assuming an unpolarised strange sea $(\Delta s=0)$ in which case the singlet and octet axial charges are identical (Eq. (20)). Taking $a_{8}$ from hyperon decay constants, they obtained $\Gamma_{1}^{p}=0.185$ and $\Gamma_{1}^{n}=-0.023$ for the proton and the neutron, respectively. Unlike the Bjorken sum rule, the Ellis--Jaffe sum rules depend on several assumptions, in particular a vanishing polarisation of strange quarks in the nucleon.

\subsection{Experimental method of the CERN experiments}

The three CERN experiments by the EMC (1985), the SMC (1992-1996) and the COMPASS Collaboration (since 2002) share the same principle. All of them use the M2 beam line providing longitudinally polarisded positive muons with momenta of up to $200 \mathrm{GeV}$. A polarisation of about $80 \%$ was measured by the $\mathrm{SMC}^{40,41}$ using two dedicated beam polarimeters. The solid-state polarised target consists of two or three cells with material of opposite polarisations, which are inverted at regular intervals. The open forward spectrometer and the polarised target are described in Sections 2.2 and 2.3 .

The experiments measure the DIS cross-section asymmetry for parallel and antiparallel orientation of muon and nucleon spins, taking advantage of the cancellation of several important quantities in the asymmetry: the dominant unpolarised cross-section, the beam flux, the number of target nuclei, and the spectrometer acceptance. From the measured DIS cross-section asymmetry the virtual-photon asymmetry

$$
A_{1}=\frac{\sigma_{\frac{1}{2}}-\sigma_{\frac{3}{2}}}{\sigma_{\frac{1}{2}}+\sigma_{\frac{3}{2}}}=\frac{g_{1}-\frac{Q^{2}}{\nu^{2}} g_{2}}{F_{1}} \rightarrow \frac{g_{1}}{F_{1}}
$$

is determined taking into account the beam and target polarisations, the fraction of polarisable nucleons in the target material and the depolarisation of the virtual photon with respect to the parent muon. Here $\sigma_{\frac{1}{2}}$ and $\sigma_{\frac{3}{2}}$ are the cross-sections for the absorption of a transversely polarised photon with spin antiparallel and parallel to the spin of the longitudinally polarised nucleon. The contribution of the structure 
function $g_{2}$ is suppressed by $Q^{2} / \nu^{2}$ and $A_{1}$ is essentially equal to the ratio of the spin-dependent and the spin-averaged structure functions $g_{1}$ and $F_{1}$.

\subsection{Experimental results}

\subsubsection{Sum rules}

The EMC proton result $\Gamma_{1}^{p}=0.126 \pm 0.010 \pm 0.015$ is in clear disagreement with the Ellis-Jaffe prediction of $0.185 \pm 0.005$. From this the EMC deduced a small axial singlet charge of $a_{0}=0.098 \pm 0.076 \pm 0.113$ and a negative strange quark contribution to the proton spin of $\Delta s+\Delta \bar{s}=-0.095 \pm 0.016 \pm 0.023 .^{34}$ Recent COMPASS results indicate a somewhat larger quark spin contribution of $a_{0}=0.33 \pm 0.03 \pm 0.05$ and a similar strange quark contribution $\Delta s+\Delta \bar{s}=$ $-0.08 \pm 0.01 \pm 0.02 .^{43}$ Still the original EMC conclusion that the quark spins do not account for most of the proton spin holds.

In 1992, the SMC performed the first measurement of the neutron $g_{1}$ structure function $^{44}$ using a polarised deuteron target and the EMC result for the proton. The measurement revealed a violation of the Ellis--Jaffe sum rule for the neutron and confirmed the Bjorken sum rule for the difference of proton and neutron first moments of $g_{1}$ (Eq. (21)). As for the deuteron, the measured $x$-range was subsequently extended also for the proton ${ }^{45}$ down to $x=0.004$ (for $Q^{2}>1 \mathrm{GeV}^{2}$ ) confirming essentially the EMC result.

Also in 1992, the ${ }^{3} \mathrm{He}$ experiment E142 at SLAC reported the contradicting findings: a validation of the Ellis-Jaffe sum rule for the neutron and thus a violation of the Bjorken sum rule. ${ }^{46}$ Due to the lower beam energy of $19 \mathrm{GeV}-26 \mathrm{GeV}$, E142 had to struggle with large QCD radiative corrections of order $\alpha_{s}\left(Q^{2}\right) / \pi$ for the Bjorken sum rule. From this $Q^{2}$ evolution Ellis and Karliner determined in 1994 the strong coupling constant $\alpha_{s}\left(M_{Z}^{2}\right)=0.122_{-0.009}^{+0.005}$ using corrections up to order $\left(\alpha_{s} / \pi\right)^{4} \cdot{ }^{47}$ Applying these corrections, the E142 result turned out to be also compatible with the Bjorken sum rule.

The most recent COMPASS result for the Bjorken integral and for the isosinglet "Ellis-Jaffe" integral $\int_{x_{\min }}^{1}\left(g_{1}^{p}+g_{1}^{n}\right) \mathrm{d} x$ is shown in Fig. 6 as a function of the lower integration limit $x_{\min }$ at $Q^{2}=3 \mathrm{GeV}^{2}$. Note that while for the Bjorken sum there is a large contribution for $x<0.1$, the contribution from this region to the Ellis-Jaffe sum is negligible. With $a_{3}=1.28 \pm 0.07 \pm 0.010$ compared to the PDG value for $\left|g_{A} / g_{V}\right|=1.2723 \pm 0.0023$ the Bjorken sum rule is confirmed at the $10 \%$ level. ${ }^{42}$

\subsubsection{Structure functions and quark helicity distributions}

The spin-dependent structure function data for the proton as obtained from the asymmetry measurements using Eq. (22) are shown in Fig. 7 as a function of $x$ and $Q^{2}$. The world data come from COMPASS, ${ }^{42,}{ }^{43} \mathrm{SMC},{ }^{48} \mathrm{EMC},{ }^{34} \mathrm{SLAC},{ }^{49-53}$ HERMES, ${ }^{54}$ and Jefferson Lab. ${ }^{55,56}$ The smallest- $x$ data were obtained by the 


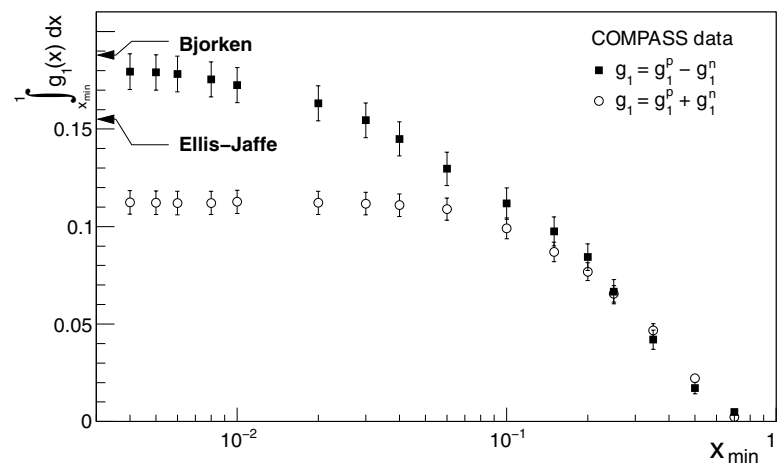

Fig. 6. Convergence of the first moments of $g_{1}^{p} \mp g_{1}^{n}$ as a function of the lower integration limit $x_{\text {min }}$ from the COMPASS proton and deuteron data. ${ }^{42}$ The arrows indicate the theoretical expectations. Error bars are statistical only.
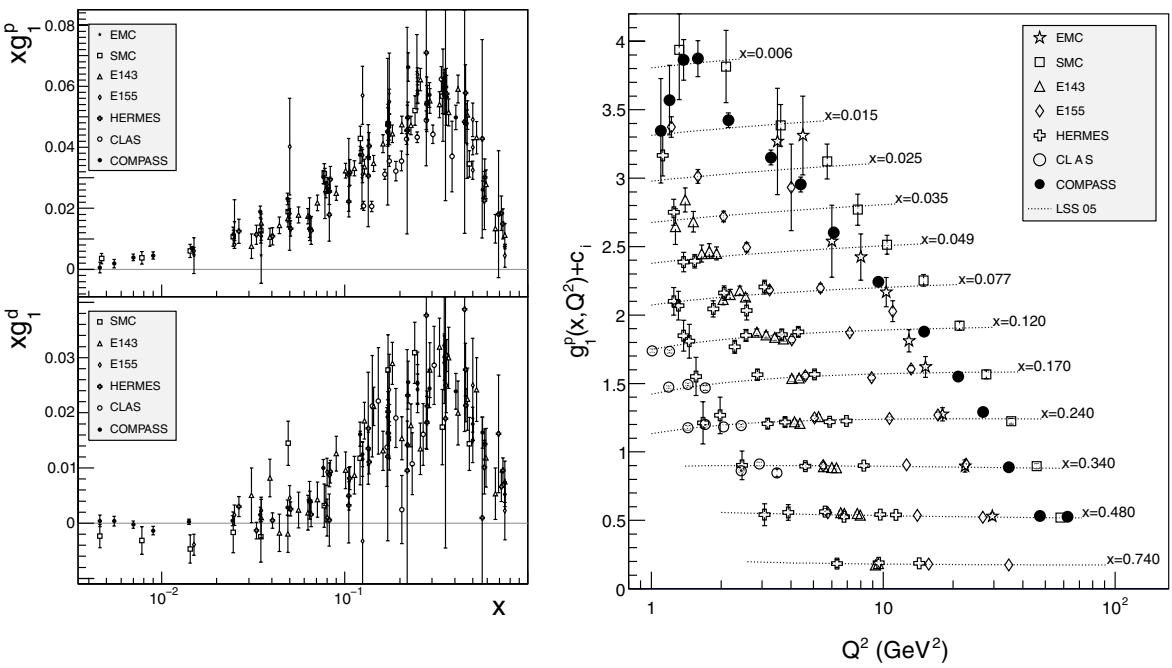

Fig. 7. Left: $x g_{1}\left(x, Q^{2}\right)$ as function of $x$ with $Q^{2}>1 \mathrm{GeV}^{2}$ for the proton (top) and the deuteron (bottom). Right: Proton data for $g_{1}\left(x, Q^{2}\right)$ as a function of $x$ and $Q^{2}$ with $W>2.5 \mathrm{GeV}$. For clarity the $g_{1}$ data for the $i$-th $x$ bin (starting from $i=0$ ) were offset by $c_{i}=0.28(11.6-i)$. Error bars are statistical errors only.

CERN experiments. Similar data exist for the deuteron. HERMES, SLAC and Jefferson Lab also obtained some neutron $\left({ }^{3} \mathrm{He}\right)$ data.

Insight into the individual quark and gluon helicity distributions can be gained from semi-inclusive deep inelastic scattering (SIDIS, Fig. 8). The probability for a quark $q$ of flavour $i$ to fragment into a hadron $h$ with energy fraction $z=E_{h} / \nu$ is described by the fragmentation function $D_{i}^{h}\left(z, Q^{2}\right)$. Due to the factorisation theorem, $x$ and $z$ dependences appear as a product of quark distribution and 


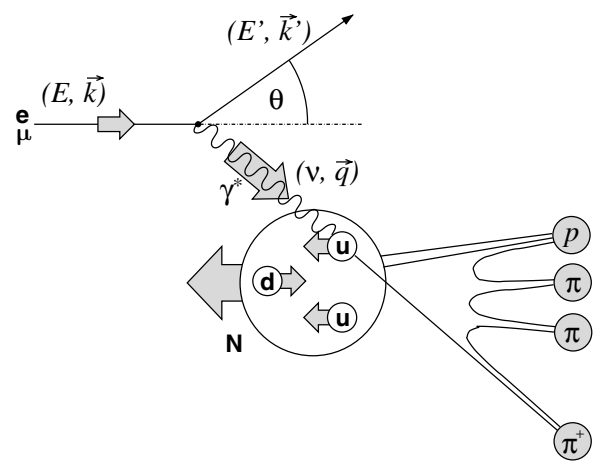

Fig. 8. Semi-inclusive deep inelastic scattering.

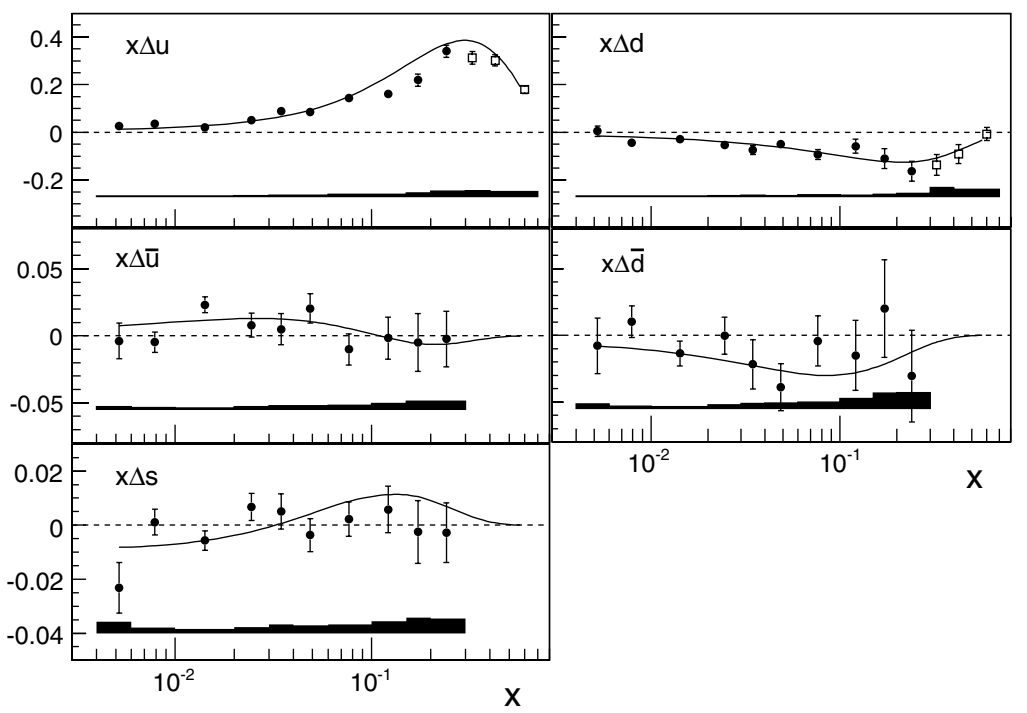

Fig. 9. Quark helicity distributions from a LO analysis. ${ }^{58}$ The bands indicate the systematic uncertainty.

fragmentation functions. Similar to the inclusive asymmetries, one obtains doublespin cross-section asymmetries for the production of a hadron $h$

$$
A_{1}^{h}\left(x, Q^{2}, z\right) \simeq \frac{\sum_{i} e_{i}^{2} \Delta q_{i}\left(x, Q^{2}\right) D_{i}^{h}\left(z, Q^{2}\right)}{\sum_{i} e_{i}^{2} q_{i}\left(x, Q^{2}\right) D_{i}^{h}\left(z, Q^{2}\right)} .
$$

An up quark preferentially fragments into a $\pi^{+}$while a down quark favours fragmenting into a $\pi^{-}$. The difference of favoured and unfavoured fragmentation allows for a flavour separation of the quark helicity distributions. The first leadingorder (LO) determination of the valence and nonstrange sea polarisation using this method was made by the SMC. ${ }^{57}$ A recent result by COMPASS is shown in Fig. 9. The up quark polarisation is positive and the one of the down quark negative. The 
strange quark polarisation is slightly positive contrary to the $x$-integrated result from the first moment of $g_{1}$. This issue is still under discussion and may be linked to the uncertainties in the fragmentation functions for strange quarks.

\subsubsection{Gluon helicity distributions}

In 1988, it was shown that the gluon polarisation contributes via the axial anomaly to the singlet axial charge like

$$
a_{0}=\sum_{q} \Delta q-3 \frac{\alpha_{s}}{2 \pi} \Delta g
$$

with $\alpha_{s} \Delta g$ constant, i.e. independent of $Q^{2} .{ }^{61,62}$ This led to the suggestion that maybe a large positive gluon polarisation would mask the quark spin contribution to the nucleon spin. In order to recover the value of 0.6 for $\Delta \Sigma$, values of $2 \hbar$ to $3 \hbar$ are required for $\Delta g$. This gave birth to the COMPASS Collaboration, which set out to determine the gluon polarisation.

The gluon polarisation can be probed in SIDIS via the gluon-photon fusion process (PGF) $\gamma g \rightarrow q \bar{q}$. Particularly interesting is the production of open charm, e.g. of $D$ mesons, because of the absence at small $x$ of charmed quarks in the nucleon. Furthermore, high- $p_{T}$ hadron pairs and single hadrons can be used to determine the gluon polarisation. The first indication that the gluon polarisation is much smaller then required by the anomaly scenario came 2005 from COMPASS ${ }^{63}$ using events with high- $p_{T}$ hadron pairs at low $Q^{2}$. Later results from open charm ${ }^{64}$ and events with high- $p_{T}$ hadron pairs at $Q^{2}>1 \mathrm{GeV}^{2}$ followed. ${ }^{65}$ The LO results of such determinations are summarised in Fig. 10. Results from RHIC confirmed the small gluon polarization, but recently also indicated that the gluon still may contribute significantly to the nucleon spin. ${ }^{66}$

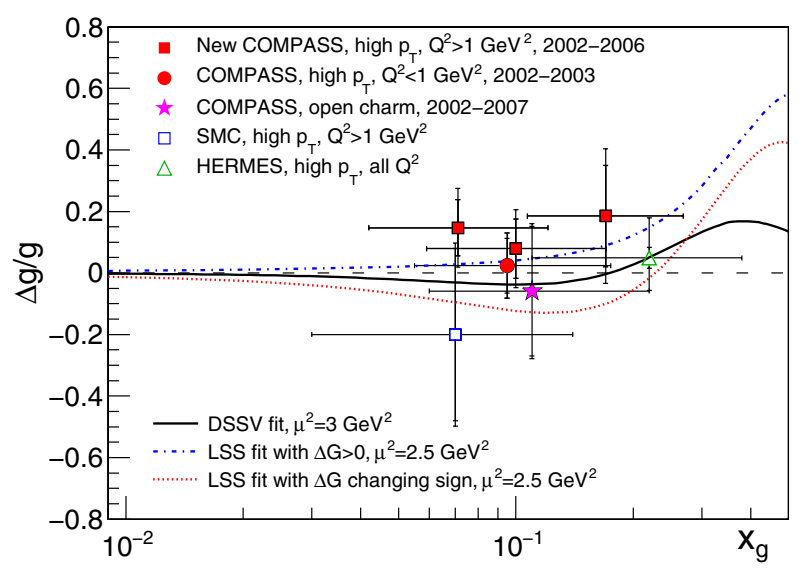

Fig. 10. Gluon polarisation $\Delta g / g$ from LO determinations as function of $x_{g}$. The horizontal error bar indicates the $x$ range of the measurement. Also shown are the results from NLO QCD analyses of the world data. ${ }^{59,} 60$ 


\subsubsection{Global QCD analyses}

Like in the spin-averaged case (see Section 3.3), the $Q^{2}$ evolution of the $g_{1}$ structure function (Fig. 7) is described by the DGLAP equations. ${ }^{25}$ From next-to-leading (NLO) QCD analyses one obtains the individual quark, antiquark and gluon helicity distributions $\Delta q\left(x, Q^{2}\right)$ and $\Delta g\left(x, Q^{2}\right)$. Modern global QCD analyses ${ }^{59,60,66}$ take into account inclusive DIS and SIDIS data as well as data from polarised $p p$ collisions at RHIC. While the quark distributions are well determined, the gluon distribution still have considerable uncertainties owing to the small $Q^{2}$ range of the data for a given $x$. A polarised electron-ion collider would change this situation dramatically as HERA did in the unpolarised case.

\subsection{Transverse spin}

\subsubsection{Transversity}

Apart from the spin-averaged $\left(F_{1}\right)$ and spin-dependent structure function $\left(g_{1}\right)$, there is at leading twist a third, chiral-odd structure function $h_{1}$ describing the distribution of transverse quark spins in a transversely polarised nucleon

$$
h_{1}(x)=\frac{1}{2} \sum_{i} e_{i}^{2} \delta q_{i}(x) \quad \text { with } \quad \delta q_{i}(x)=q_{i}^{\uparrow}(x)-q_{i}^{\downarrow}(x),
$$

where $q^{\uparrow}$ and $q^{\downarrow}$ respectively indicate the number densities of quarks with spin orientation parallel and antiparallel to the transverse nucleon spin. In the nonrelativistic case $h_{1}(x)$ is equal to $g_{1}(x)$. This structure function does not contribute to inclusive scattering, because it implies a flip of the quark spin, which is conserved for massless quarks. However, in SIDIS $h_{1}$ can be coupled to the chirally odd Collins fragmentation function $\Delta_{T} D_{i}^{h}\left(z, p_{T}\right)$ and thus lead to an azimuthal sine modulation of the cross-section asymmetry in the Collins angle ${ }^{\mathrm{b}} \phi_{\mathrm{Coll}}=\phi_{h}+\phi_{S}+\pi$ with an amplitude of

$$
A_{\text {Coll }}(x, z) \sim \frac{\sum_{i} e_{i}^{2} \delta q_{i}(x) \Delta_{T} D_{i}^{h}\left(z, p_{T}^{h}\right)}{\sum_{q} e_{i}^{2} q_{i}(x) D_{i}^{h}\left(z, p_{T}^{h}\right)} .
$$

Here $p_{T}^{h}$ denotes the transverse hadron momentum with respect to the virtual photon; $\phi_{h}$ and $\phi_{S}$ are the azimuthal angles of the hadron and the nucleon spin. Figure 11 (top) shows the Collins asymmetry for the proton as measured for positive and negative hadrons (dominantly pions). Similar measurements exist for identified pions and kaons ${ }^{67}$ and from HERMES. The corresponding asymmetries for the deuteron are compatible with zero due to a cancellation of the up and down quark contributions.

Transversity can — instead of to the Collins function - also couple to another chiral-odd fragmentation function, the interference fragmentation function (IFF),

\footnotetext{
${ }^{\mathrm{b}}$ Note that some experiments, e.g. HERMES, use a definition of $\phi_{\text {Coll }}$ without adding $\pi$.
} 

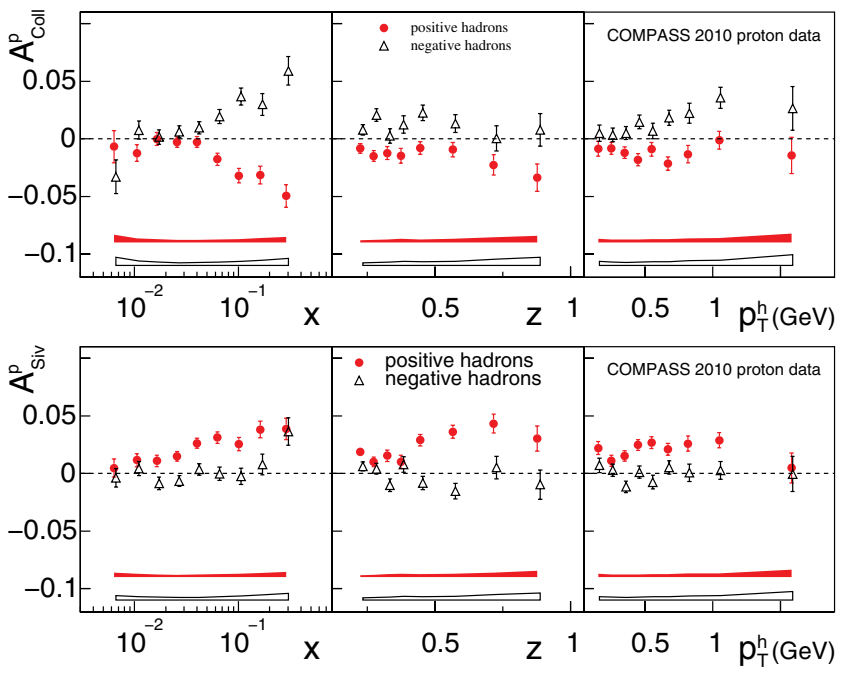

Fig. 11. Collins (top) and Sivers (bottom) asymmetry 68,69 of the proton for positive and negative hadrons as functions of $x, z$, and $p_{T}^{h}$ from COMPASS. The bands indicate the systematic uncertainty.

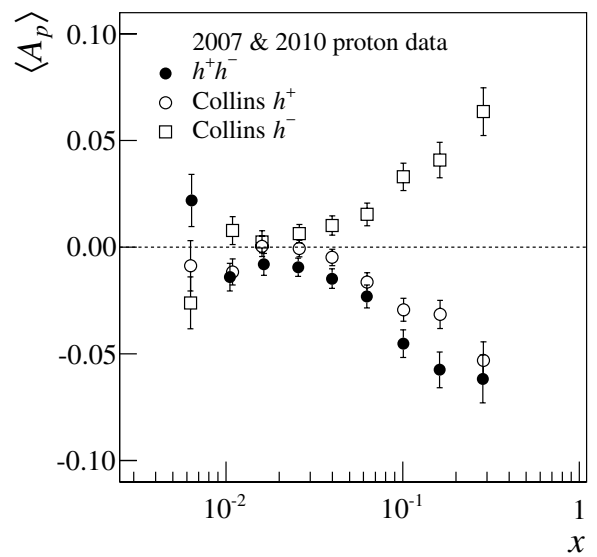

Fig. 12. Collins asymmetries for positive (lower open circles) and negative hadrons (upper open circles) and 2-hadron asymmetries (full circles) as function of $x .^{68,70}$

which generates a pair of oppositely charged hadrons. The similarity of the Collins asymmetry (for positive hadrons) and the 2-hadron asymmetry suggests that a common mechanism is at work in both cases (Fig. 12).

Phenomenological determinations of the transversity structure function ${ }^{71}$ have been made using data from COMPASS, HERMES, and Belle. At Belle, the Collins and IFF fragmentation function have been measured in $e^{+} e^{-}$collisions. These 
analyses show a positive transverse polarisation of the up quarks and a negative one for down quarks in a transversely polarised nucleon.

\subsubsection{Transverse-momentum-dependent parton distributions}

The PDFs discussed so far do not explain the strong transverse single-spin asymmetries observed in both hadron and DIS reactions indicating significant spin-orbit coupling in the nucleon associated with the quark transverse momentum $k_{T}$. Transverse-momentum-dependent (TMD) distributions allow for such a $k_{T}$ dependence in addition to the one on the longitudinal momentum fraction $x$ carried by the parton. In QCD there are eight leading-twist transverse-momentumdependent parton distributions listed below.

\begin{tabular}{|c|c|c|c|}
\hline $\mathrm{N}$ & $\mathrm{U}$ & $\mathrm{L}$ & $\mathrm{T}$ \\
\hline $\mathrm{U}$ & $\boldsymbol{f}_{\mathbf{1}}$ & & $h_{1}^{\perp}$ \\
\hline $\mathrm{L}$ & & $\boldsymbol{g}_{\mathbf{1}}$ & $h_{1 \mathrm{~L}}^{\perp}$ \\
\hline $\mathrm{T}$ & $f_{1 \mathrm{~T}}^{\perp}$ & $g_{1 \mathrm{~T}}^{\perp}$ & $\boldsymbol{h}_{\mathbf{1}} h_{1 \mathrm{~T}}^{\perp}$ \\
\hline
\end{tabular}

Here U, L, and T stand for unpolarised, longitudinally polarised, and transversely polarised nucleons (rows) and quarks (columns), respectively. Upon integration over $k_{T}$ the TMD PDFs in the diagonal (in bold) yield the usual structure functions $F_{1}(x), g_{1}(x)$, and $h_{1}(x)$, while all off-diagonal TMD PDFs vanish.

The best known TMD PDF is the Sivers function $f_{1 \mathrm{~T}}^{\perp}$ which describes the distribution of unpolarised quarks in a transversely polarised nucleon. It couples to the standard unpolarised fragmentation function $D_{i}^{h}$ and causes an azimuthal asymmetry in $\sin \phi_{\text {Siv }}$, where the Sivers angle is defined by $\phi_{\text {Siv }}=\phi_{h}-\phi_{S}$. Figure 11 (bottom) shows the Sivers asymmetry of the proton for positive and negative hadrons. For positive hadrons a clear positive asymmetry is visible, in particular at larger $x$ and $z$. The Boer-Mulders function $h_{1}^{\perp}$ describes the distribution of transversely polarised quarks in an unpolarised nucleon and couples to the Collins fragmentation function. As the Sivers function, the Boer-Mulders function is odd under time reversal and only nonvanishing due to initial or final-state interactions. For these functions only a restricted universality is predicted implying a change of sign when going from SIDIS to Drell-Yan (DY) reactions

$$
\left.f_{1 \mathrm{~T}}^{\perp}\right|_{\mathrm{SIDIS}}=-\left.f_{1 \mathrm{~T}}^{\perp}\right|_{\mathrm{DY}} \quad \text { and }\left.\quad h_{1}^{\perp}\right|_{\mathrm{SIDIS}}=-\left.h_{1}^{\perp}\right|_{\mathrm{DY}}
$$

An experimental test of this prediction is an important next step in spin physics.

COMPASS has the unique possibility to measure with the same spectrometer the sign change of the T-odd Sivers and Boer-Mulders PDFs in the upcoming, first-ever polarised Drell-Yan experiment with a pion beam planned for 2015 . 


\subsubsection{Generalised parton distributions}

The role of the orbital angular momentum in the nucleon is still unclear and the only known access to this quantity is via generalised parton distribution functions (GPD),${ }^{72}$ which correlate longitudinal momentum and transverse spatial degrees of freedom. They enter in the cross-sections for deeply virtual Compton scattering and hard exclusive meson production. COMPASS started to study theses processes and in 2016/17 will have GPD runs with a $2.5 \mathrm{~m}$ long liquid hydrogen target.

\section{Conclusions}

Deep inelastic muon scattering at the SPS has been a unique success story for more than 35 years now, and has grown into the most long-lived experimental programme of CERN. It has had a fundamental impact on the present-day understanding of the innermost structure of hadronic matter, and has been an important and fruitful testing ground for the Quark-Parton Model and for Quantum Chromodynamics, which it helped establishing as the universally accepted theory of the strong interaction of quarks and gluons. It has even outlived the HERA electron-proton collider programme at DESY, which had some of its major roots in the CERN muon experiments.

The question whether deep inelastic lepton scattering has a future after COMPASS and HERA cannot be answered today. Only the discovery of a substructure of quarks or leptons would warrant the investment in a major new programme; by colliding protons from the LHC - or a future hadron collider of even higher energy - with a new, high-energy electron beam, CERN would be well equipped to repeat the successes of its deep inelastic scattering programmes at a new energy frontier.

\section{References}

1. W. Panofsky, Electromagnetic Interactions: Low $q^{2}$ Electrodynamics: Elastic and Inelastic Electron (and Muon) Scattering, in Proc. 14th Int. Conf. on High-Energy Physics, Vienna (1968).

2. M. Gell-Mann, A Schematic Model of Baryons and Mesons, Phys. Lett. 8, 214-215 (1964). doi: 10.1016/S0031-9163(64)92001-3.

3. G. Zweig, An SU(3) model for strong interaction symmetry and its breaking (Version 2), CERN-TH-412 (1964).

4. J. Aubert et al., The ratio of the nucleon structure functions $F_{2}$ for iron and deuterium, Phys. Lett. B 123, 275 (1983). doi: 10.1016/0370-2693(83)90437-9.

5. R. Clifft and N. Doble, Proposed Design of a High-Energy, High Intensity Muon Beam for the SPS North Experimental Area (1974), CERN/LAB. II/EA/74-2, CERN/SPSC/74-12. 
6. J. Aubert et al., A Large Magnetic Spectrometer System for High-Energy Muon Physics, Nucl. Instrum. Meth. 179, 445-466 (1981). doi: 10.1016/0029$554 \mathrm{X}(81) 90169-5$.

7. J. Aubert et al., A Detailed Study of the Proton Structure Functions in Deep Inelastic Muon-Proton Scattering, Nucl. Phys. B 259, 189 (1985). doi: 10.1016/05503213(85)90635-2.

8. J. Albanese et al., The Vertex and Large Angle Detectors of a Spectrometer System for High-energy Muon Physics, Nucl. Instrum. Meth. 212, 111 (1983). doi: 10.1016/01675087(83)90682-8.

9. D. Bollini et al., A High Luminosity Spectrometer for Deep Inelastic Muon Scattering Experiments, Nucl. Instrum. Meth. 204, 333 (1983). doi: 10.1016/01675087(83)90063-7.

10. A. Benvenuti et al., An Upgraded Configuration of a High Luminosity Spectrometer for Deep Inelastic Muon Scattering Experiments, Nucl. Instrum. Meth. A 226, 330 (1984). doi: 10.1016/0168-9002(84)90045-7.

11. P. Amaudruz et al., Precision measurement of the structure function ratios $F_{2}(\mathrm{He}) / F_{2}(\mathrm{D}), F_{2}(\mathrm{C}) / F_{2}(\mathrm{D})$ and $F_{2}(\mathrm{Ca}) / F_{2}(\mathrm{D})$, Z. Phys. C 51, 387-394 (1991). doi: $10.1007 / \mathrm{BF} 01548560$.

12. P. Abbon et al., The COMPASS experiment at CERN, Nucl. Instrum. Meth. A 577, 455-518 (2007). doi: 10.1016/j.nima.2007.03.026.

13. P. Abbon et al., The COMPASS Setup for Physics with Hadron Beams, hepex/1410.1797 (2014).

14. A. Argento et al., Electroweak Asymmetry in Deep Inelastic Muon-Nucleon Scattering, Phys. Lett. B 120, 245 (1983). doi: 10.1016/0370-2693(83)90665-2.

15. A. Argento et al., Measurement of the Interference Structure Function $x g_{3}(x)$ in Muon-Nucleon Scattering, Phys. Lett. B 140, 142, (1984). doi: 10.1016/03702693(84)91065-7.

16. F. Halzen and A. D. Martin, Quarks and Leptons (Wiley, 1984). ISBN 9780471887416.

17. R. Roberts, The Structure of the Proton: Deep Inelastic Scattering (Cambridge Monographs on Mathematical Physics), (Cambridge University Press, 1990).

18. E. D. Bloom et al., High-Energy Inelastic e-p Scattering at 6 Degrees and 10 Degrees, Phys. Rev. Lett. 23, 930-934 (1969). doi: 10.1103/PhysRevLett.23.930.

19. M. Breidenbach et al., Observed Behavior of Highly Inelastic Electron-Proton Scattering, Phys. Rev. Lett. 23, 935-939 (1969). doi: 10.1103/PhysRevLett.23.935.

20. J. Bjorken, Asymptotic Sum Rules at Infinite Momentum, Phys. Rev. 179, 1547-1553 (1969). doi: 10.1103/PhysRev.179.1547.

21. A. Benvenuti et al., A High Statistics Measurement of the Proton Structure Functions $F_{2}\left(x, Q^{2}\right)$ and $R$ from Deep Inelastic Muon Scattering at High $Q^{2}$, Phys. Lett. B 223, 485 (1989). doi: 10.1016/0370-2693(89)91637-7.

22. M. Arneodo et al., Measurement of the proton and the deuteron structure functions $F_{2}^{p}$ and $F_{2}^{d}$, Phys. Lett. B 364, 107-115 (1995). doi: 10.1016/0370-2693(95)01318-9.

23. L. Whitlow, E. Riordan, S. Dasu, S. Rock, and A. Bodek, Precise measurements of the proton and deuteron structure functions from a global analysis of the SLAC deep inelastic electron scattering cross-sections, Phys. Lett. B 282, 475-482 (1992). doi: 10.1016/0370-2693(92)90672-Q.

24. M. Adams et al., Proton and deuteron structure functions in muon scattering at 470 GeV, Phys. Rev. D 54, 3006-3056 (1996). doi: 10.1103/PhysRevD.54.3006. 
25. G. Altarelli and G. Parisi, Asymptotic Freedom in Parton Language, Nucl. Phys. B 126, 298 (1977). doi: 10.1016/0550-3213(77)90384-4.

26. A. Benvenuti et al., Test of QCD and a Measurement of $\Lambda$ From Scaling Violations in the Nucleon Structure Function $F_{2}\left(x, Q^{2}\right)$ at High $Q^{2}$, Phys. Lett. B 195, 97 (1987). doi: 10.1016/0370-2693(87)90892-6.

27. A. Benvenuti et al., Test of QCD and a Measurement of $\Lambda$ From Scaling Violations in the Proton Structure Function $F_{2}\left(x, Q^{2}\right)$ at High $Q^{2}$, Phys. Lett. B 223, 490 (1989). doi: 10.1016/0370-2693(89)91638-9.

28. A. Benvenuti et al., A High Statistics Measurement of the Deuteron Structure Functions $F_{2}\left(x, Q^{2}\right)$ and $R$ From Deep Inelastic Muon Scattering at High $Q^{2}$, Phys. Lett. B 237, 592 (1990). doi: 10.1016/0370-2693(90)91231-Y.

29. M. Virchaux and A. Milsztajn, A Measurement of $\alpha_{s}$ and higher twists from a QCD analysis of high statistics $F_{2}$ data on hydrogen and deuterium targets, Phys. Lett. $B$ 274, 221-229 (1992). doi: 10.1016/0370-2693(92)90527-B.

30. R. K. Ellis, W. Furmanski, and R. Petronzio, Unraveling Higher Twists, Nucl. Phys. B 212, 29 (1983). doi: 10.1016/0550-3213(83)90597-7.

31. S. I. Alekhin, Combined analysis of SLAC-BCDMS-NMC data at high $x: \alpha_{s}$ and high twists, hep-ph/9907350 (1999).

32. K. Olive et al. (Review of Particle Physics), Chin. Phys. C 38, 090001 (2014). doi: 10.1088/1674-1137/38/9/090001.

33. J. Ashman et al., A Measurement of the Spin Asymmetry and Determination of the Structure Function $g_{1}$ in Deep Inelastic Muon-Proton Scattering, Phys. Lett. B 206, 364 (1988). doi: 10.1016/0370-2693(88)91523-7.

34. J. Ashman et al., An Investigation of the Spin Structure of the Proton in Deep Inelastic Scattering of Polarized Muons on Polarized Protons, Nucl. Phys. B 328, 1 (1989). doi: 10.1016/0550-3213(89)90089-8.

35. E. Leader and M. Anselmino, A Crisis in the Parton Model: Where, Oh Where Is the Proton's Spin?, Z. Phys. C 41, 239 (1988). doi: 10.1007/BF01566922.

36. C. A. Aidala et al., The Spin Structure of the Nucleon, Rev. Mod. Phys. 85, 655-691 (2013). doi: 10.1103/RevModPhys.85.655.

37. J. Bjorken, Applications of the Chiral U(6) $\times(6)$ Algebra of Current Densities, Phys. Rev. 148, 1467-1478 (1966). doi: 10.1103/PhysRev.148.1467.

38. J. Bjorken, Inelastic Scattering of Polarized Leptons from Polarized Nucleons, Phys. Rev. D 1, 1376-1379 (1970). doi: 10.1103/PhysRevD.1.1376.

39. J. R. Ellis and R. L. Jaffe, A Sum Rule for Deep Inelastic Electroproduction from Polarized Protons, Phys. Rev. D 9, 1444 (1974). doi: 10.1103/PhysRevD.10.1669.2, 10.1103/PhysRevD.9.1444.

40. B. Adeva et al., Measurement of the polarization of a high-energy muon beam, Nucl. Instrum. Meth. A 343, 363-373 (1994). doi: 10.1016/0168-9002(94)90213-5.

41. D. Adams et al., Measurement of the SMC muon beam polarization using the asymmetry in the elastic scattering off polarized electrons, Nucl. Instrum. Meth. A 443, 1-19 (2000). doi: 10.1016/S0168-9002(99)01017-7.

42. M. Alekseev et al., The Spin-dependent Structure Function of the Proton $g_{1}^{p}$ and a Test of the Bjorken Sum Rule, Phys. Lett. B 690, 466-472 (2010). doi: 10.1016/j.physletb.2010.05.069.

43. V. Y. Alexakhin et al., The Deuteron Spin-dependent Structure Function $g_{1}^{d}$ and its First Moment, Phys. Lett. B 647, 8-17, (2007). doi: 10.1016/j.physletb.2006.12.076. 
44. B. Adeva et al., Measurement of the spin dependent structure function $g_{1}(x)$ of the deuteron, Phys. Lett. B 302, 533-539 (1993). doi: 10.1016/0370-2693(93)90438-N.

45. D. Adams et al., Measurement of the spin dependent structure function $g_{1}(x)$ of the proton, Phys. Lett. B 329, 399-406 (1994). doi: 10.1016/0370-2693(94)90793-5.

46. P. Anthony et al., Determination of the neutron spin structure function, Phys. Rev. Lett. 71, 959-962 (1993). doi: 10.1103/PhysRevLett.71.959.

47. J. R. Ellis and M. Karliner, Determination of $\alpha_{S}$ and the nucleon spin decomposition using recent polarized structure function data, Phys. Lett. B 341, 397-406 (1995). doi: 10.1016/0370-2693(95)80021-O.

48. B. Adeva et al., Spin asymmetries $A_{1}$ and structure functions $g_{1}$ of the proton and the deuteron from polarized high-energy muon scattering, Phys. Rev. D 58, 112001 (1998). doi: 10.1103/PhysRevD.58.112001.

49. P. Anthony et al., Deep inelastic scattering of polarized electrons by polarized He-3 and the study of the neutron spin structure, Phys. Rev. D 54, 6620-6650 (1996). doi: 10.1103/PhysRevD.54.6620.

50. K. Abe et al., Precision determination of the neutron spin structure function $g_{1}^{n}$, Phys. Rev. Lett. 79, 26-30 (1997). doi: 10.1103/PhysRevLett.79.26.

51. K. Abe et al., Measurements of the proton and deuteron spin structure functions $g_{1}$ and $g_{2}$, Phys. Rev. D 58, 112003, (1998). doi: 10.1103/PhysRevD.58.112003.

52. P. Anthony et al., Measurement of the deuteron spin structure function $g_{1}^{d}(x)$ for $1(\mathrm{GeV} / c)^{2}<Q^{2}<40(\mathrm{GeV} / c)^{2}$, Phys. Lett. B 463, 339-345 (1999). doi: 10.1016/S0370-2693(99)00940-5.

53. P. Anthony et al., Measurements of the $Q^{2}$ dependence of the proton and neutron spin structure functions $g_{1}^{p}$ and $g_{1}^{n}$, Phys. Lett. B 493, 19-28 (2000). doi: 10.1016/S03702693(00)01014-5.

54. A. Airapetian et al., Precise determination of the spin structure function $g_{1}$ of the proton, deuteron and neutron, Phys. Rev. D 75, 012007 (2007). doi: 10.1103/PhysRevD.75.012007.

55. X. Zheng et al., Precision measurement of the neutron spin asymmetry $A_{1}^{N}$ and spin flavor decomposition in the valence quark region, Phys. Rev. Lett. 92, 012004 (2004). doi: 10.1103/PhysRevLett.92.012004.

56. K. Dharmawardane et al., Measurement of the $x$ - and $Q^{2}$-dependence of the asymmetry $A_{1}$ on the nucleon, Phys. Lett. B 641, 11-17 (2006). doi: 10.1016/j. physletb.2006.08.011.

57. B. Adeva et al., Polarization of valence and nonstrange sea quarks in the nucleon from semiinclusive spin asymmetries, Phys. Lett. B 369, 93-100 (1996). doi: 10.1016/03702693(95)01584-1.

58. M. Alekseev et al., Quark helicity distributions from longitudinal spin asymmetries in muon-proton and muon-deuteron scattering, Phys. Lett. B 693, 227-235 (2010). doi: 10.1016/j.physletb.2010.08.034.

59. D. de Florian et al., Extraction of Spin-Dependent Parton Densities and Their Uncertainties, Phys. Rev. D 80, 034030 (2009). doi: 10.1103/PhysRevD.80.034030.

60. E. Leader, A. V. Sidorov, and D. B. Stamenov, Determination of Polarized PDFs from a QCD Analysis of Inclusive and Semi-inclusive Deep Inelastic Scattering Data, Phys. Rev. D 82, 114018 (2010). doi: 10.1103/PhysRevD.82.114018. 
61. G. Altarelli and G. G. Ross, The Anomalous Gluon Contribution to Polarized Leptoproduction, Phys. Lett. B 212, 391 (1988). doi: 10.1016/0370-2693(88)91335-4.

62. A. Efremov and O. Teryaev, Spin Structure of the Nucleon and Triangle Anomaly, Nucl. Phys. (1988).

63. E. Ageev et al., Gluon polarization in the nucleon from quasi-real photoproduction of high- $p_{T}$ hadron pairs, Phys. Lett. $B$ 633, 25-32 (2006). doi: 10.1016/j.physletb.2005.11.049.

64. C. Adolph et al., Leading and Next-to-Leading Order Gluon Polarization in the Nucleon and Longitudinal Double Spin Asymmetries from Open Charm Muoproduction, Phys. Rev. D 87 (5), 052018, (2013). doi: 10.1103/PhysRevD.87.052018.

65. C. Adolph et al., Leading order determination of the gluon polarisation from DIS events with high- $p_{T}$ hadron pairs, Phys. Lett. B 718, 922-930 (2013). doi: 10.1016/j.physletb.2012.11.056.

66. D. de Florian et al., Evidence for polarization of gluons in the proton, Phys. Rev. Lett. 113, 012001 (2014). doi: 10.1103/PhysRevLett.113.012001.

67. C. Adolph et al. Collins and Sivers asymmetries in muon production of pions and kaons off transversely polarised proton, hep-ex/1408.4405 (2014).

68. C. Adolph et al., Experimental investigation of transverse spin asymmetries in muon$p$ SIDIS processes: Sivers asymmetries, Phys. Lett. B 717, 383-389 (2012). doi: 10.1016/j.physletb.2012.09.056.

69. C. Adolph et al., Experimental investigation of transverse spin asymmetries in muon$p$ SIDIS processes: Collins asymmetries, Phys. Lett. B 717, 376-382 (2012). doi: 10.1016/j.physletb.2012.09.055.

70. C. Adolph et al., A high-statistics measurement of transverse spin effects in dihadron production from muon-proton semi-inclusive deep inelastic scattering, Phys. Lett. B 736, 124-131 (2014). doi: 10.1016/j.physletb.2014.06.080.

71. M. Anselmino et al., Simultaneous extraction of transversity and Collins functions from new SIDIS and $e^{+} e^{-}-$data, Phys. Rev. D 87, 094019 (2013). doi: 10.1103/PhysRevD.87.094019.

72. X.-D. Ji, Gauge-Invariant Decomposition of Nucleon Spin, Phys. Rev. Lett. 78, 610-613 (1997). doi: 10.1103/PhysRevLett.78.610. 